IDETC2018- 85539

\title{
EXPERIMENTAL AND THEORETICAL STUDY OF TWO-TO-ONE INTERNAL RESONANCE OF MEMS RESONATORS
}

\author{
Amal Z. Hajjaj \\ Physical Science and Engineering Division \\ King Abdullah University of Science and Technology \\ Thuwal, Makah Province, Saudi Arabia \\ Amal.Hajjaj@kaust.edu.sa
}

\author{
Feras K. Alfosail \\ Physical Science and Engineering Division \\ King Abdullah University of Science and Technology \\ Thuwal, Makah Province, Saudi Arabia \\ Feras.Alfosail@kaust.edu.sa
}

\author{
Mohammad I. Younis \\ Physical Science and Engineering Division \\ King Abdullah University of Science and Technology \\ Thuwal, Makah Province, Saudi Arabia \\ Mohammad.Younis@kaust.edu.sa
}

\begin{abstract}
In this paper, we investigate experimentally and theoretically the two-to-one (2:1) internal resonance between the first two symmetric vibrational modes of microelectromechanical (MEMS) arch resonator electrothermally tuned and electrostatically driven. Applying electrothermal voltage across the beam anchors controls its stiffness and then its resonance frequencies. Hence the ratio between the two frequencies can be tuned to a ratio of two. Then, we study the dynamic response of the arch beam during internal resonance. In the studied case, the presence of high AC bias excitation leads to the direct simultaneous excitation of the $1^{\text {st }}$ and $3^{\text {rd }}$ frequencies in addition to the activation of the internal resonance. A reduced order model and perturbation techniques are presented to analyze the nonlinear response of the structure. In the perturbation technique, the direct excitation of the $3 r d$ resonance frequency is taken into consideration. Results show the presence of Hopf bifurcations, which can lead to chaotic motion at higher excitation. A good agreement among the theoretical and experimental results is shown.
\end{abstract}

Keywords: Arch MEMS Resonator, Electrothermal Actuation, Electrostatic Actuation, Internal resonance, Direct Excitation, Perturbation Techniques

\section{INTRODUCTION}

The nonlinear interaction among various modes of vibration of micro/nanoelectromechanical structures (M/NEMS) has been widely investigated in recent years [1-8]. This is motivated by the fact that the mode coupling in M/NEMS resonators is vital for successful implementation of these devices in various applications. The two major mechanisms of mode coupling that have been reported and proposed in significant applications in the literature are mainly veering [1-4] and internal resonance [5-9].

Internal resonance or auto-parametric resonance denotes the exchange of energy between two vibrational modes in a structure briefly. These modes can be either in the same or a different plane of vibration. One necessary condition to activate internal resonance is that the ratio between the two involved modes is an integer. The most common internal resonances investigated in the literature are mostly the 1:1, 2:1, and 3:1. The internal resonance has been investigated in several structures in the literature [5-14].

The dynamic and the static behavior of initially curved MEMS resonators have been extensively investigated [15-17]. These structures have attracted many researcher thanks to their different and interesting characteristics: bi-stability, snapthrough motion, and a wide range of nonlinearity (cubic from the stretching and quadratic from the initial curvature). Moreover, one of the interesting characteristics of the arch 
structures is that its first vibrational mode increases as varying its stiffness and its third mode decreases, which allows a wide range of ratios among the various modes. This makes the curved structures possess several internal resonances $(1: 1,2: 1$, $3: 1$, and $4: 1$ ). The $3: 1$ and $1: 1$ internal resonances of clampedclamped curved structures under electrostatic force have been extensively studied numerically and experimentally [11-14].

Despite the extensive research on internal resonance on M/NEMS resonators, there is lack of integrated and comprehensive theoretical and experimental investigation on the topic. This work aims to study the two-to-one internal resonances of an initially curved (arch) beam experimentally and theoretically. We investigate experimentally and theoretically; using reduced order model (ROM) and multiple scale technique (MTS); the 2:1 internal resonance between the $1^{\text {st }}$ and $3^{\text {rd }}$ vibrational mode of an arch beam electrothermally tuned and electrostatically driven. The electrothermal actuation, in this case, is used to control the stiffness of the arch beam. In MTS, the influence of the high AC bias voltage is accounted for by considering the direct simultaneous excitation of the two participating modes.

The rest of the paper is organized as follows. The experimental setup is presented in Section 2. The dynamic response of the arch beam is modeled using the nonlinear EulerBernoulli beam equation combined with the heat conduction equation and is solved in Section 3. The perturbation method is described in Section 4. A discussion of the dynamic response of the arch beam near internal resonance is presented in Section 5. Finally, the main conclusions are summarized in Section 6.

\section{EXPERIMENTAL SETUP}

We conducted our experiments on fabricated arch beams fabricated by MEMCAP [18], from SOI wafers with highly conductive $\mathrm{Si}$ device layer using two mask process. The stroboscopic video microscopy from Polytec [19] was used to study and investigate the free vibration and the frequency response of the resonator.

The arch beam under consideration, Fig. 1, is tuned electrothermally and driven electrostatically. By applying a DC voltage, $V_{T H}$, between the anchors of the arch beam, a DC current $I_{T H}$ passes through it heating it up, which controls its induced axial load. The arch beam is separated from a stationary electrode with a transduction gap width $d$. The curved beam is actuated electrostatically by a DC bias voltage $V_{D C}$ and an AC harmonic voltage of amplitude $V_{A C}$ and frequency $\hat{\Omega}$.

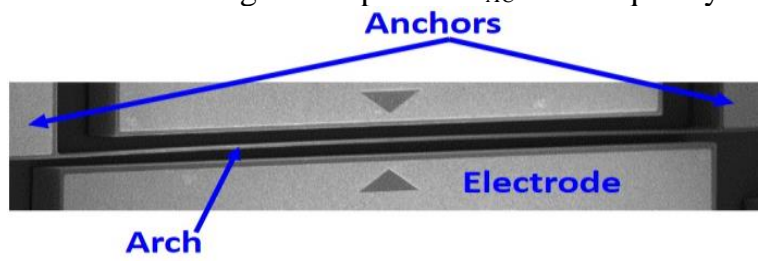

(a)

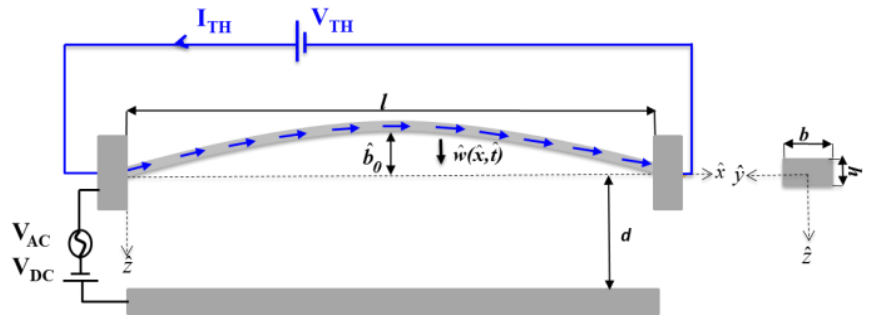

(b)

Figure 1: (a) An SEM image of the arch beam (b) Schematic of the clampedclamped arch beam electrothermally and electrostatically actuated.

\section{Problem Formulation}

The device under consideration consists of a curved clamped-clamped beam of an initial shape $\hat{w}_{0}(\hat{x})$ governed by

$\hat{w}_{0}(\hat{x})=\left(\hat{b}_{0}-R+\sqrt{R^{2}-(\hat{x}-l / 2)^{2}}\right)(u[1+\hat{x}]-u[\hat{x}])$

where $u(\hat{x})$ and $R$ represent the Heaviside function and the radius of the arc, respectively, $\hat{x}$ is the position along the microbeam, and $\hat{b}_{0}$ is the rise at the mid-point of the arch.

The governing equation of motion of the curved beam under consideration describing its transverse deflection $\hat{w}(\hat{x}, \hat{t})$, where $\hat{t}$ is the time, is written as follows [20-21]:

$$
\begin{aligned}
& \rho b h \frac{\partial^{2} \hat{w}}{\partial \hat{t}^{2}}+E I \frac{\partial^{4} \hat{w}}{\partial \hat{x}^{4}}+\hat{c} \frac{\partial \hat{w}}{\partial \hat{t}}=
\end{aligned}
$$

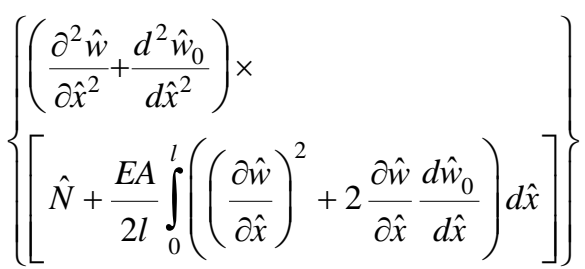

$$
\begin{aligned}
& +\frac{1}{2} \varepsilon b \frac{\left(V_{D C}+V_{A C} \cos (\hat{\Omega} t)\right)^{2}}{\left(d-\hat{w}-\hat{w}_{0}\right)^{2}}
\end{aligned}
$$

The arch beam is subjected to the below boundary conditions

$$
\hat{w}(0, \hat{t})=\hat{w}(l, \hat{t})=0 \text { and }\left.\frac{\partial \hat{w}}{\partial \hat{x}}\right|_{(0, \hat{t})}=\left.\frac{\partial \hat{w}}{\partial \hat{x}}\right|_{(l, \hat{t})}=0
$$

The arch beam has Young's modulus $E$, material density $\rho$, length $l$, width $b$, and thickness $h$. The cross-section area is assumed rectangular with $A=b h$ with a moment of inertia given by $I=b h^{3} / 12$. The curved beam is subjected to viscous damping of coefficient $\hat{c}$. The parameter $\varepsilon$ represents the dielectric constant of the medium. The term $\hat{N}=\hat{N}_{0}-\hat{S}_{T h}$ describes the tensile axial load, where $\hat{N}_{0}$ is arising from the fabrication process and $\hat{S}_{T h}$ denotes the thermal compressive load given by 


$$
\hat{S}_{T h}=\frac{E A}{l} \int_{0}^{l} \alpha(T)\left(T[\hat{x}]-T_{a}\right) d \hat{x}
$$

In Eq. (4), $T[\hat{x}]$ denotes the average temperature along the curved beam induced by $I_{T H} . T[\hat{x}]$ is computed using Fourier's law by the procedure prescribed in [2]. The parameter $\alpha(T)$ is the thermal expansion coefficient, which is assumed dependent on temperature.

For convenience, we introduce the nondimensional variables:

$w=\frac{\hat{w}}{d} ; x=\frac{\hat{x}}{l} ; t=\frac{\hat{t}}{T_{S}} ; w_{0}=\frac{\hat{w}_{0}}{d} ;$ and $b_{0}=\frac{\hat{b}_{0}}{d}$

where $T_{S}=\sqrt{\rho b h l^{4} / E I}$ is the timescale. Substituting Eq. (5) into Eq. (2) and Eq. (3), we obtain the nondimensional equation of motion of the beam

$$
\begin{aligned}
& \frac{\partial^{2} w}{\partial t^{2}}+\frac{\partial^{4} w}{\partial x^{4}}+c \frac{\partial w}{\partial t}= \\
& \left(\left(\frac{\partial^{2} w}{\partial x^{2}}+\frac{d^{2} w_{0}}{d x^{2}}\right)\left[N+\alpha_{1} \int_{0}^{1}\left[\frac{\partial w^{2}}{\partial x}+2 \frac{\partial w}{\partial x} \frac{d w_{0}}{d x}\right] d x\right]\right) \\
& +\alpha_{2} \frac{\left(V_{D C}+V_{A C} \cos (\Omega t)\right)^{2}}{\left(1-w-w_{0}\right)^{2}}
\end{aligned}
$$

subjected to the nondimensional boundary conditions

$w(0, t)=w(1, t)=0$ and $\left.\frac{\partial w}{\partial x}\right|_{(0, t)}=\left.\frac{\partial w}{\partial x}\right|_{(1, t)}=0$

The parameters appearing in Eq. (6) are defined as

$$
\begin{aligned}
& \alpha_{1}=6 \frac{d^{2}}{h^{2}} ; \quad \mathrm{N}=N_{0}+S_{T H} ; \quad N_{0}=\frac{l^{2}}{E I} \hat{N}_{0} ; \\
& S_{T H}=\frac{l^{2}}{E I} \hat{S}_{T H} ; \quad \alpha_{2}=\frac{6 \varepsilon l^{4}}{E h^{3} d^{3}} ; \\
& c=\frac{l^{4}}{E I T} \hat{c} ; \Omega=T \hat{\Omega}
\end{aligned}
$$

The eigenvalue problem of the arch beam under consideration is solved following the general procedure described in [2]. Furthermore, we simulate the dynamic response of the arch beam under electrostatic force and at a constant electrothermal voltage by discretizing Eq. (6) using the Galerkin procedure, which yields a reduced order model (ROM). Then, the transverse deflection of the arch beam is written as [21]:

$$
w(x, t)=\sum_{i=0}^{n} \mathrm{u}_{i}(t) \phi_{i}(x)
$$

where $\mathrm{u}_{i}(t)(i=0 \ldots n)$ are the nondimensional modal coordinates and $\phi_{i}(x)(i=0 \ldots n)$ are the mode shapes of the arch beam at a constant electrothermal voltage [2].
Following [2, 20-21], we cross-multiply Eq. (6) to reduce the computational costs. Then, by substituting Eq. (9) into Eq. (6), multiplying by $\phi_{j}(x)$ and integrating along the arch beam, this yields $n$ algebraic equations regarding $\mathrm{u}_{i}(t)$

$$
\begin{aligned}
& \sum_{i=0}^{n} M_{i j} \ddot{u}_{i}(t)+\sum_{i=0}^{n} C_{i j} \dot{u}_{i}(t)+\sum_{i=0}^{n} K_{i j} u_{i}(t)=F c_{j}+F e_{j}(t) \\
& \forall(j=0 \ldots n)
\end{aligned}
$$

where

$$
\begin{aligned}
& M_{i j}=\int_{0}^{1}\left[\phi_{i}(x) \phi_{j}(x)\left(1-w(x, t)-w_{0}(x)\right)^{2}\right] d x \\
& C_{i j}=c \int_{0}^{1}\left[\phi_{i}(x) \phi_{j}(x)\left(1-w(x, t)-w_{0}(x)\right)^{2}\right] d x \\
& K_{i j}=\int_{0}^{1}\left[\phi_{i}^{(i v)}(x) \phi_{j}(x)\left(1-w(x, t)-w_{0}(x)\right)^{2}\right] d x \\
& F e_{j}(t)=\alpha_{2}\left(V_{D C}+V_{A C} \operatorname{Cos}(\Omega t)\right)^{2} \int_{0}^{1} \phi_{j}(x) d x \\
& F c_{j}=\alpha_{1} \Gamma \int_{0}^{1}\left[\phi_{j}(x)\left(\sum_{i=0}^{n} u_{i}(t) \phi_{i}{ }^{\prime \prime}(x)+w_{0}{ }^{\prime \prime}(x)\right)\left(1-w(x, t)-w_{0}(x)\right)^{2}\right] d x \\
& \Gamma=N+\int_{0}^{1}\left(\sum_{i=0}^{n} u_{i}(t) \phi_{i}^{\prime}(x)\right)^{2} d x+2 \int_{0}^{1}\left(\sum_{i=0}^{n} u_{i}(t) \phi_{i}^{\prime}(x)\right) w_{0}{ }^{\prime}(x) d x
\end{aligned}
$$

To solve the dynamic response of the arch beam given by Eq. (10), we compute the values of the integrals in Eq. (11). Then, a set of differential equations is obtained and integrated in time using Runge-Kutta technique and by using four symmetric mode shapes [2].

\section{PERTURBATION ANALYSIS}

In this section, we consider analyzing the two-to-one (2:1) internal resonance using the method of multiple scales (MTS) [22] in Eq. (6).

The expansion of the electrostatic forcing term $\left(V_{D C}+V_{A C} \cos (\Omega t)\right)^{2}$ results into

$$
\begin{gathered}
\left(V_{D C}+V_{A C} \cos (\Omega t)\right)^{2}= \\
V_{E f f D C}{ }^{2}+\left\{2 V_{A C} V_{D C} \cos (\Omega t)+\frac{1}{2} V_{A C}{ }^{2} \cos (2 \Omega t)\right\} \\
V_{E f f D C}{ }^{2}=V_{D C}{ }^{2}+\frac{1}{2} V_{A C}{ }^{2}
\end{gathered}
$$

where $V_{E f f D C}$ is the effective electrostatic voltage and the last term in the right handside of Eq. (12) represents the harmonic excitations. Moreover, the activation of the (2:1) internal resonance in the presence of the simultaneous excitation of $\Omega$ 
and $2 \Omega$ terms can trigger the two resonance cases. Then, we seek a third order expansion of the form

$w(x, t ; \varepsilon)=\sum_{n=1}^{3} \varepsilon^{n} w_{n}\left(x, T_{0}, T_{1}, T_{2}\right)$

where $\varepsilon$ is introduced as a bookkeeping parameter, $T_{i}=\varepsilon^{i} t$. The scale chosen at Eq. (14) determines that the quadratic nonlinearity is at $\varepsilon^{2}$ and the cubic nonlinearity is at $\varepsilon^{3}$ and the forcing and damping are assumed to be $\varepsilon^{2} \cos (\Omega t)$, $\varepsilon^{2} \cos (2 \Omega t)$ and $\varepsilon c$, respectively, while the electrostatic forcing term is expanded following the procedure outlined in [23]. We substitute Eq. (14) into Eq. (6) and expanding the electrostatic force yield

Order $\varepsilon$

$$
\begin{gathered}
\ell\left(w_{1}\right)=D_{0}{ }^{2} w_{1}+ \\
w_{1}^{i v}-N w_{1}^{\prime \prime}-2 \alpha_{1} w_{s}^{\prime \prime}\left(\int_{0}^{1} w_{s}^{\prime} w_{1}^{\prime} \mathrm{d} x\right) \\
-\frac{2 \alpha_{2} V_{E f f D C}}{\left(1-w_{s}\right)^{3}} w_{1}=0
\end{gathered}
$$

$\operatorname{Order} \varepsilon^{2}$

$$
\begin{aligned}
\ell\left(w_{2}\right)= & -2 D_{0} D_{1} w_{1}-c D_{0} w_{1}+\alpha_{1} w_{s}^{\prime \prime}\left(\int_{0}^{1}\left(w_{1}^{\prime}\right)^{2} \mathrm{~d} x\right) \\
& +2 \alpha_{1} w_{1}^{\prime \prime}\left(\int_{0}^{1} w_{s}^{\prime} w_{1}^{\prime} \mathrm{d} x\right)+\frac{3 \alpha_{2} V_{E f f D C}}{\left(1-w_{s}\right)^{4}} w_{1}^{2} \\
& +\frac{2 \alpha_{2} V_{A C} V_{D C}}{\left(1-w_{s}\right)^{2}} \cos \left(\Omega T_{0}\right)+\frac{\alpha_{2} V_{A C}^{2}}{2\left(1-w_{s}\right)^{2}} \cos \left(2 \Omega T_{0}\right)
\end{aligned}
$$

Order $\varepsilon^{3}$

$$
\begin{aligned}
\ell\left(w_{3}\right)= & -2 D_{0} D_{2} w_{1}-2 D_{0} D_{1} w_{2}-D_{1}^{2} w_{1}-c D_{1} w_{1}-c D_{0} w_{2} \\
& +\frac{4 \alpha_{2} V_{A C} V_{D C}}{\left(1-w_{s}\right)^{3}} \cos \left(T_{0} \Omega\right) w_{1}+\frac{\alpha_{2} V_{A C}^{2}}{\left(1-w_{s}\right)^{3}} \cos \left(2 \Omega T_{0}\right) w_{1} \\
& +\frac{6 \alpha_{2} V_{E f D C}}{\left(1-w_{s}\right)^{4}} w_{1} w_{2}+2 \alpha_{1} w_{s}^{\prime \prime}\left(\int_{0}^{1} w_{1}^{\prime} w_{2}^{\prime} \mathrm{d} x\right) \\
& +2 \alpha_{1} w_{2}^{\prime \prime}\left(\int_{0}^{1} w_{s}^{\prime} w_{1}^{\prime} \mathrm{d} x\right)+2 \alpha_{1} w_{1}^{\prime \prime}\left(\int_{0}^{1} w_{s}^{\prime} w_{2}^{\prime} \mathrm{d} x\right) \\
& +\alpha_{1} w_{1}^{\prime}\left(\int_{0}^{1}\left(w_{1}^{\prime}\right)^{2} \mathrm{~d} x\right)
\end{aligned}
$$

where the derivatives are defined as $D_{j}{ }^{i}=\frac{\partial^{i}}{\partial T_{j}{ }^{i}} \quad$ and ( )' denotes the derivative with respect to $x$. The solution to Eq. (15) is given by the linear eigenvalue problem [2]. To activate the (2:1) internal resonance we assume the excitation frequency to be around the first primary resonance, i.e., $\Omega=\omega_{m}+\varepsilon \sigma_{1}$ and the second natural frequency to be $\omega_{n}=2 \omega_{m}+\varepsilon \sigma_{2}$, where $\sigma_{1}$ and $\sigma_{2}$ are detuning parameters. The (2:1) internal resonance is activated at the second order $\varepsilon^{2}$ and we carry out the expansion up to the third order $\varepsilon^{3}$ to capture the influence of cubic nonlinearity induced by mid-plane stretching and obtain the solvability condition following the work in [24]. The final outcome of this process after separating the real and the imaginary parts are four modulation equations governing the amplitude and phase relation, which are

$$
\begin{aligned}
& a_{m}^{\prime}=-\frac{\mu_{1}}{2} a_{m}-a_{n} \sin \left(\beta_{m}-\beta_{n}\right)\left(\frac{F_{3 V D C}}{2 \omega_{m}}+\frac{R_{1} F_{2 V D C}}{8 \omega_{m}^{3}}\right) \\
& -\frac{R_{1}}{4 \omega_{m}} a_{m} a_{n} \sin \left(2 \beta_{m}-\beta_{n}\right)-\left(\frac{F_{3 V A C}}{2 \omega_{m}}+\frac{R_{1} F_{2 V A C}}{8 \omega_{m} \omega_{n}^{2}}\right) a_{m} \sin \left(2 \beta_{m}\right) \\
& +a_{m} a_{n} \cos \left(2 \beta_{m}-\beta_{n}\right)\left(\frac{\mu_{1} R_{1}}{8 \omega_{m}^{2}}-\frac{\mu_{2} R_{1}}{16 \omega_{m} \omega_{n}}\right)+\frac{\mu_{1} F_{2 V D C}}{4 \omega_{m}^{2}} \cos \left(\beta_{m}\right) \\
& -\frac{F_{2 V D C}}{\omega_{m}} \sin \left(\beta_{m}\right) \\
& a_{m} \beta_{m}{ }^{\prime}=-\left(\frac{\mu_{1}^{2}}{8 \omega_{m}}+\sigma_{1}\right) a_{m}-\frac{\mu_{1} F_{2 V D C}}{4 \omega_{m}^{2}} \sin \left(\beta_{m}\right)-\frac{F_{2 V D C}}{\omega_{m}} \cos \left(\beta_{m}\right) \\
& -\left(\frac{F_{3 V D C}}{2 \omega_{m}}+\frac{R_{1} F_{2 V D C}}{8 \omega_{m}^{3}}\right) a_{n} \cos \left(\beta_{m}-\beta_{n}\right)-\left(\frac{K_{1}}{8 \omega_{m}}+\frac{R_{1}^{2}}{32 \omega_{m}^{3}}\right) a_{m} a_{n}^{2} \\
& +\left(\frac{\mu_{2} R_{1}}{16 \omega_{m} \omega_{n}}-\frac{\mu_{1} R_{1}}{8 \omega_{m}^{2}}\right) a_{m} a_{n} \sin \left(2 \beta_{m}-\beta_{n}\right) \\
& -\left(\frac{K_{2}}{8 \omega_{m}}+\frac{R_{1} R_{2}}{32 \omega_{m} \omega_{n}^{2}}\right) a_{m}^{3}-\frac{R_{1}}{4 \omega_{m}} a_{m} a_{n} \cos \left(2 \beta_{m}-\beta_{n}\right) \\
& -a_{m} \cos \left(2 \beta_{m}\right)\left(\frac{F_{3 V A C}}{2 \omega_{m}}+\frac{R_{1} F_{2 V A C}}{8 \omega_{m} \omega_{n}^{2}}\right) \\
& a_{n}^{\prime}=-\frac{\mu_{2}}{2} a_{n}+\left(\frac{R_{2} F_{2 V D C}}{4 \omega_{m}^{2} \omega_{n}}+\frac{F_{3 V D C}}{2 \omega_{n}}\right) a_{m} \sin \left(\beta_{m}-\beta_{n}\right) \\
& +\left(\frac{\mu_{2} R_{2}}{16 \omega_{n}^{2}}-\frac{\mu_{1} R_{2}}{8 \omega_{m} \omega_{n}}\right) a_{m}^{2} \cos \left(2 \beta_{m}-\beta_{n}\right) \\
& +\frac{R_{2}}{4 \omega_{n}} a_{m}^{2} \sin \left(2 \beta_{m}-\beta_{n}\right)+\frac{\mu_{2} F_{2 V A C}}{4 \omega_{n}^{2}} \cos \left(\beta_{n}\right)-\frac{F_{2 V A C}}{\omega_{n}} \sin \left(\beta_{n}\right) \\
& a_{n} \beta_{n}^{\prime}=\left(\sigma_{2}-2 \sigma_{1}-\frac{\mu_{2}^{2}}{8 \omega_{n}}\right) a_{n}-\left(\frac{F_{3 V D C}}{2 \omega_{n}}+\frac{R_{2} F_{2 V D C}}{4 \omega_{m}^{2} \omega_{n}}\right) a_{m} \cos \left(\beta_{m}-\beta_{n}\right) \\
& -\frac{K_{4}}{8 \omega_{n}} a_{n}^{3}-\left(\frac{K_{3}}{8 \omega_{n}}+\frac{R_{1} R_{2}}{16 \omega_{m}^{2} \omega_{n}}\right) a_{m}^{2} a_{n}-\frac{R_{2}}{4 \omega_{n}} a_{m}^{2} \cos \left(2 \beta_{m}-\beta_{n}\right) \\
& +\left(\frac{\mu_{2} R_{2}}{16 \omega_{n}^{2}}-\frac{\mu_{1} R_{2}}{8 \omega_{m} \omega_{n}}\right) a_{m}^{2} \sin \left(2 \beta_{m}-\beta_{n}\right)-\frac{\mu_{2} F_{2 V A C}}{4 \omega_{n}^{2}} \sin \left(\beta_{n}\right) \\
& -\frac{F_{2 V A C}}{\omega_{n}} \cos \left(\beta_{n}\right)
\end{aligned}
$$

where $a_{m}$ and $a_{n}$ are the amplitudes of the first and second primary resonances while $\beta_{m}$ and $\beta_{n}$ are the phases corresponding to each mode, respectively. The coefficients $\mu_{i}, F_{i}, R_{i}$ and $K_{i}$ correspond to damping, forcing, second order coupling terms and third order coupling terms, respectively, and the definition of each coefficient is in 
appendix A. Equation (18) to Eq. (21) are solved using arc length continuation technique to obtain the equilibrium fixed points. In addition, the stability of each fixed point is studied by examining the eigenvalues of the Jacobian of the system in complex Cartesian form. Finally, the final solution of the system is expressed as

$$
\begin{aligned}
w & =w_{s}+a_{m} \phi_{m}(x) \cos \left(\Omega t+\beta_{m}\right)+\frac{\mu_{1}}{4 \omega_{m}} a_{m} \phi_{m}(x) \sin \left(\Omega t+\beta_{m}\right) \\
& +a_{n} \phi_{n}(x) \cos \left(2 \Omega t+\beta_{n}\right)+\frac{\mu_{2}}{4 \omega_{n}} a_{n} \phi_{n}(x) \sin \left(2 \Omega t+\beta_{n}\right) \\
& +\frac{a_{n}^{2}}{2} \psi_{2}(x) \cos 4\left(2 \Omega t+2 \beta_{n}\right)+\frac{a_{n}^{2}}{2} \psi_{4}(x)+\frac{a_{m}^{2}}{2} \psi_{3}(x) \\
& +\frac{a_{m} a_{n}}{2} \psi_{1}(x) \cos \left(3 \Omega t+\beta_{m}+\beta_{n}\right)+\frac{\bar{F}_{2 V D C}}{2 \omega_{m}^{2}} \phi_{m}(x) \cos (\Omega t) \\
& +\frac{R_{2}}{8 \omega_{n}^{2}} a_{m}^{2} \phi_{n}(x) \cos \left(2 \Omega t+2 \beta_{m}\right)+\frac{\bar{F}_{2 V A C}}{2 \omega_{n}^{2}} \phi_{n}(x) \cos (2 \Omega t) \\
& +\frac{R_{1}}{8 \omega_{m}^{2}} a_{m} a_{n} \phi_{m}(x) \cos \left(\Omega t+\beta_{n}-\beta_{m}\right)
\end{aligned}
$$

\section{Results and Discussion}

For the current study, the dimensions of the arch under consideration are given in Table 1. As tuning the electrothermal voltage, the arch beam heats up, and then its stiffness increases. Fig. 2(a) shows the variation of the first two symmetric resonance frequencies of the arch beam under consideration while tuning the electrothermal voltage, experimentally and analytically. Increasing the compressive load increases the first resonance frequency while the third resonance frequency decreases then starts to increase when the first resonance frequency seems to begin to flatten (veering) [2]. Computing the ratio between both frequencies, Fig. 2(b), reveals that different internal resonances can be activated. In this study, we focus on the nonlinear aspects of the two-to-one internal resonance.

TABLE 1. GEOMETRICAL PROPERTIES OF THE MICROBEAM MADE OF DOPED SILICON.

\begin{tabular}{|c|c|c|c|c|}
\hline $\begin{array}{c}\text { Length, } l \\
(\boldsymbol{\mu m})\end{array}$ & $\begin{array}{c}\text { Thickness, } h \\
(\boldsymbol{\mu m})\end{array}$ & $\begin{array}{c}\text { Width, } b \\
(\boldsymbol{\mu m})\end{array}$ & $\begin{array}{c}\text { Gap, } d \\
(\boldsymbol{\mu m})\end{array}$ & $\begin{array}{c}\text { Initial rise, } \hat{b}_{0} \\
(\boldsymbol{\mu m})\end{array}$ \\
\hline 800 & 1.75 & 30 & 8.4 & 2.6 \\
\hline
\end{tabular}

To investigate the dynamics of the 2:1 internal resonance, we experimentally sweep the frequency for different electrostatic forces around both the first and third resonance frequencies and constant electrothermal voltage $V_{T h}=2.45 \mathrm{~V}$, Fig. 3. The experiments were conducted in atmospheric pressure (high damping) requiring high $\mathrm{AC}$ harmonic excitation amplitudes to activate the internal resonance. At lower AC excitations, the system yields a linear response, Fig. 3(a). However, increasing the electrostatic force gradually results into the splitting of the vibration amplitude into two peaks where the arch beam starts to experience internal resonance, Fig. 3(a). The presence of two peaks suggests a nonlinear modal interaction among the first and third modes.

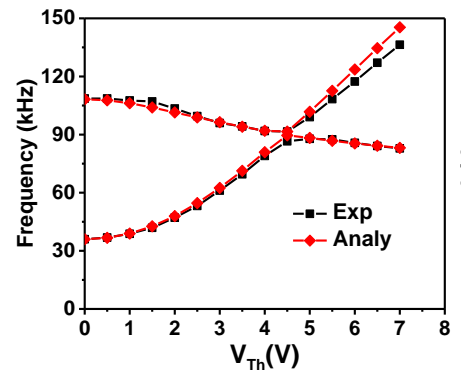

(a)

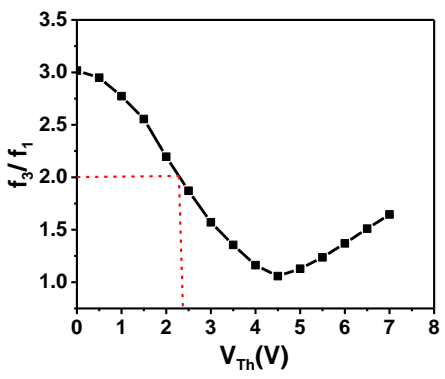

(b)
Figure 2: (a) The variation of the the first and third resonance frequency with the electrothermal voltage. (b) The variation of the ratio between both frequencies with $V_{T h}$.

On the other hand, we excite the arch beam, for the same $V_{T H}$, around its third resonance frequency for different electrostatic forces, Fig. 3(b). As increasing the forcing, the arch beam starts to experience internal resonance, which was demonstrated by the splitting of the frequency response curve. The separation between the two existing peaks increases with exaction. For larger forcing, a potential coexistence of states is shown in Fig. 3(c) (the red scatters).

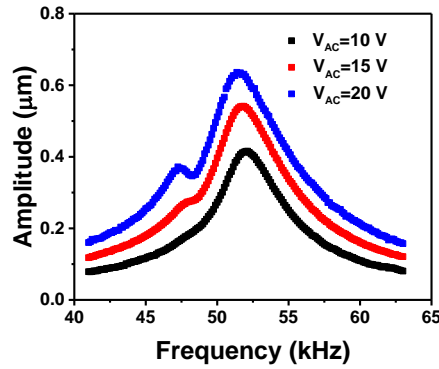

(a)

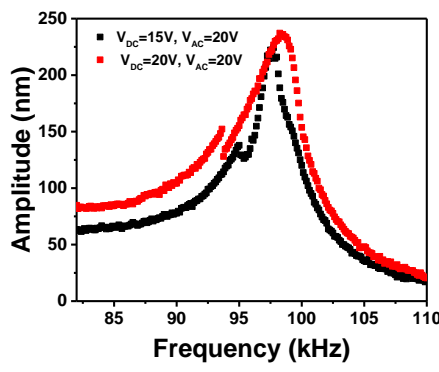

(c)

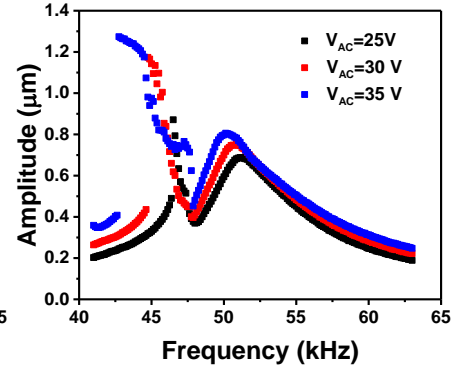

(b)

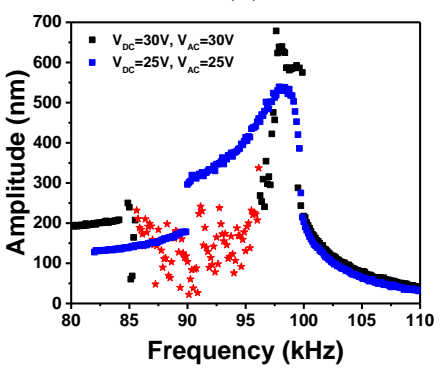

(d)
Figure 3: (a) and (b) Measured frequency responses around the first resonance frequency at $V_{T h}=2.45 \mathrm{~V}, \mathrm{~V}_{\mathrm{DC}}=15 \mathrm{~V}$, and for different AC bias voltages. (c) and (d) Measured frequency responses around the third resonance frequency at $V_{T h}=2.45 \mathrm{~V}$ for different electrostatic forces.

Due to the limitations of the experimental setup, we use the method of (MTS) and numerical simulation of the Galerkin model to analyze the nonlinear interactions involved, around the first mode, Figs. 4 and 5. A good agreement is shown among the theoretical and experimental results. In addition, since the 
forcing amplitude term $\frac{1}{2} V_{A C}{ }^{2}$ values can reach order of magnitudes similar to that of the $2 V_{A C} V_{D C}$ term in Eq. (12), the influence of the $2 \Omega$ term cannot be neglected when exciting the first primary resonance. Perturbation results indicate that the level of nonlinear interaction is influenced by the simultaneous excitation of the two modes as well as the internal resonance.

Next, we study the stability of the system via perturbation method. As we sweep the frequency, Fig. 5(c), the system experiences a saddle-node (SN) bifurcation from the lower periodic solution to the upper solution. Then, the response of the system passes through a forward and reverse Hopf bifurcations where a new limit cycle is created. Then, another saddle-node bifurcation is observed to the right lower state where the response is periodic. The behavior of the limit cycle can be observed and analyzed in the dynamical response of the system using the Galerkin method.

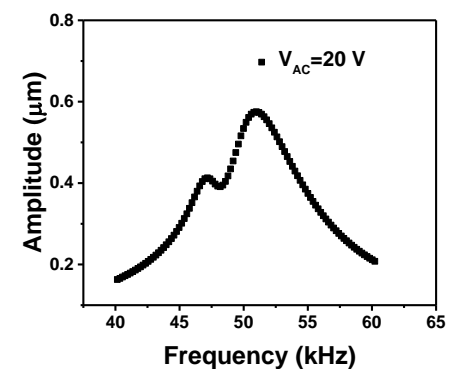

(a)

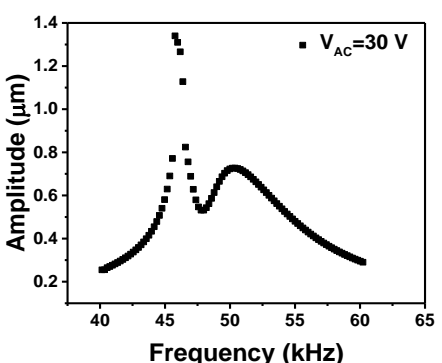

(b)

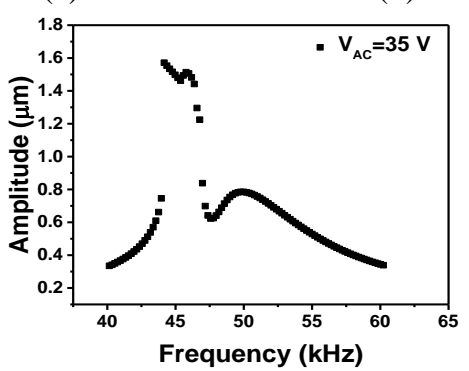

(c)

Figure 4: The Galerkin simulations of the dynamic response of the arch beam at $V_{T h}=2.45 \mathrm{~V}, V_{D C}=15 \mathrm{~V}$ and (a) $V_{A C}=20 \mathrm{~V}$, (b) $V_{A C}=30 \mathrm{~V}$, and (c) $V_{A C}=35 \mathrm{~V}$

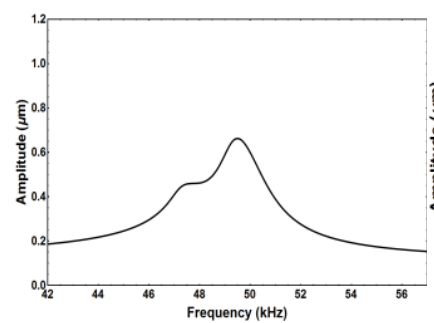

(a)

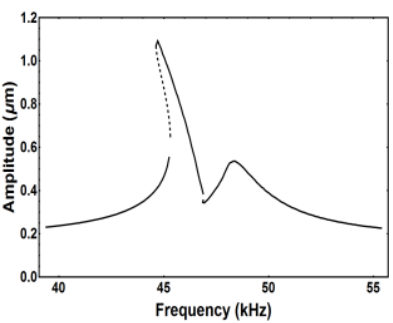

(b)

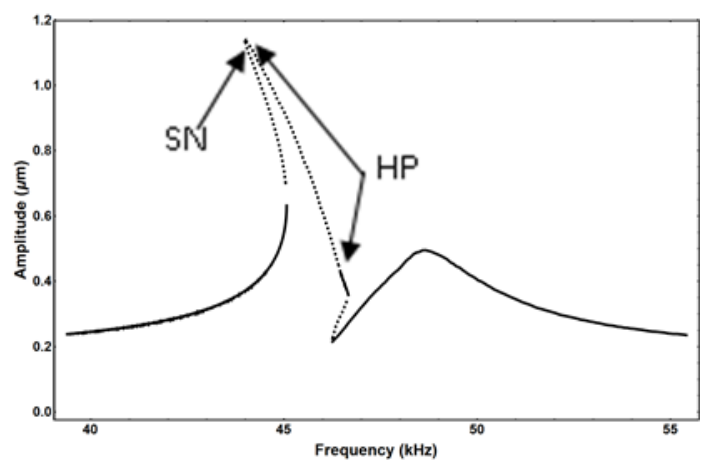

(c)

Figure 5: The perturbation simulations of the dynamic response of the arch beam at $V_{T h}=2.45 \mathrm{~V}, V_{D C}=15 \mathrm{~V}$ and (a) $V_{A C}=20 \mathrm{~V}$, (b) $V_{A C}=30 \mathrm{~V}$, and (c) $V_{A C}=35 \mathrm{~V}$. The solid line presents the stable branches. The dashed lines show the unstable branches.

We further increase the AC bias voltage, which leads to more complicated dynamic phenomena. Fig. 6(a) shows different jumps to some unstable points (discrete red) in the frequency response curve. The vibration videos at these frequencies show irratic motions that are aperiodic.

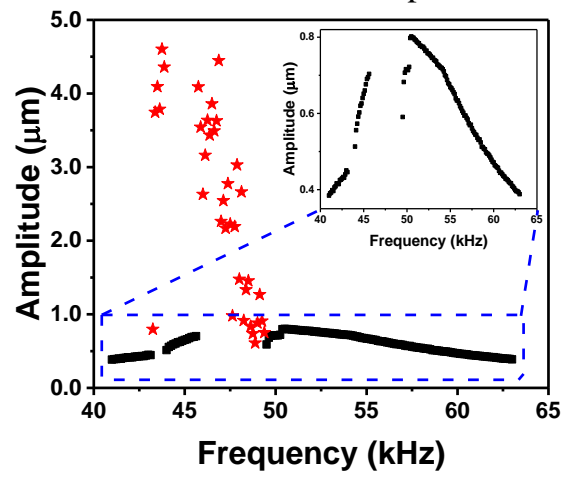

(a)

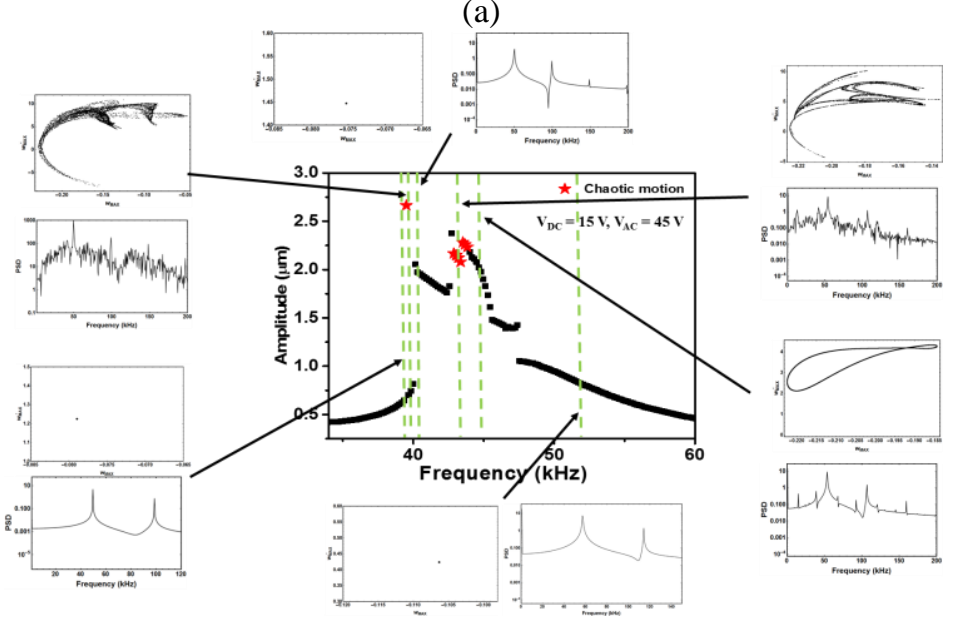

(b)

Figure 6: (a) and (b) Experimental (middle figure) and numerical results of the dynamic response of the arch beam at $V_{T h}=2.45 \mathrm{~V}, V_{D C}=15 \mathrm{~V}$, and $V_{A C}=35 \mathrm{~V}$, respectively. The red stars denotes the aperiodic (potentially chaotic) motion.

To investigate the nature of the dynamic response, we simulate the response of the arch beam at higher AC bias voltage, Fig. 6(b). This shows that even far from the internal 
resonance interaction, at $39 \mathrm{kHz}$, two contributing frequencies exist: the first and the third, that are produced due to the direct excitation at high AC bias voltage. Then, as sweeping the frequency, the response jumps from the periodic to aperiodic solutions, presented by red points. Poincare sections at these frequencies show the presence of a chaotic motion that might describe the strange motion observed experimentally. In between those chaotic solutions, the response emerges from aperiodic to periodic and then aperiodic solution, which consists of the contribution of both frequencies. Next, the system passes through a quasi-periodic motion, from $44.75 \mathrm{kHz}$ to $45.4 \mathrm{kHz}$, which might be a consequence of a reverse Hopf bifurcation. More investigation is needed to explore the dynamic response and identify either this aperiodic motion is originated only from the mode coupling of both modes via internal resonance or from the combination of internal resonance and direct excitation of the third mode due to high AC bias voltage.

\section{Conclusions}

In this paper, we investigated the 2:1 internal resonance considering the influence of the direct excitation of the third resonance frequency of an arch resonator. The arch beam was actuated electrothermally to control its stiffness and hence control the ratio between the first two symmetric vibration modes. As exciting the arch beam electrostatically at a constant electrothermal voltage, when the ratio between both frequencies equals to two, we investigated the dynamic response and the activation of the internal resonance. We showed that at high AC bias voltage, a chaotic motion might appear. Theoretically, we solved the dynamic response of the arch beam using two techniques: long time integration and perturbation technique. We added a new correction in the perturbation technique to account for the direct excitation of the third mode. The results showed a good agreement among the different methods. A future perspective of the two-to-one internal resonance similar to the observed in this paper can be realized in various application, such as chaotic computation [25] and sensing.

\section{Acknowledgment:}

This research was support by KAUST.

\section{References}

[1] A.Z. Hajjaj, M.A. Hafiz, M.I. Younis, Mode Coupling and Nonlinear Resonances of MEMS Arch Resonators for Bandpass Filters, Scientific Reports, 7 (2017) 41820.

[2] A.Z. Hajjaj, N. Alcheikh, M.I. Younis, The static and dynamic behavior of MEMS arch resonators near veering and the impact of initial shapes, International Journal of Non-Linear Mechanics, 95 (2017) 277-286.

[3] H.M. Ouakad, M.I. Younis, Natural frequencies and mode shapes of initially curved carbon nanotube resonators under electric excitation, Journal of Sound and Vibration, 330 (2011) 3182-3195.

[4] V. Sazonova, Y. Yaish, H. Üstünel, D. Roundy, T.A. Arias, P.L. McEuen, A tunable carbon nanotube electromechanical oscillator, Nature, 431 (2004) 284-287.

[5] A.H. Ramini, A.Z. Hajjaj, M.I. Younis, Tunable Resonators for Nonlinear Modal Interactions, Scientific Reports, 6 (2016).

[6] R. Karabalin, M. Cross, M. Roukes, Nonlinear dynamics and chaos in two coupled nanomechanical resonators, Physical Review B, 79 (2009) 165309.

[7] C. Lan, W. Qin, W. Deng, Energy harvesting by dynamic unstability and internal resonance for piezoelectric beam, Applied Physics Letters, 107 (2015) 093902.

[8] W.J. Venstra, R. van Leeuwen, H.S. van der Zant, Strongly coupled modes in a weakly driven micromechanical resonator, Applied Physics Letters, 101 (2012) 243111.

[9] D. Antonio, D.H. Zanette, D. López, Frequency stabilization in nonlinear micromechanical oscillators, Nature communications, 3 (2012) 806.

[10] D. Jiang, C. Pierre, S. Shaw, The construction of non-linear normal modes for systems with internal resonance, International Journal of Non-Linear Mechanics, 40 (2005) 729-746.

[11] A.H. Nayfeh, Nonlinear interactions: analytical, computational and experimental methods, Wiley, 2000.

[12] A.H. Nayfeh, W. Lacarbonara, C.-M. Chin, Nonlinear normal modes of buckled beams: three-to-one and one-to-one internal resonances, Nonlinear Dynamics, 18 (1999) 253-273.

[13] H.M. Ouakad, H.M. Sedighi, M.I. Younis, One-to-one and three-to-one internal resonances in MEMS shallow arches, Journal of Computational and Nonlinear Dynamics, 12 (2017) 051025.

[14] N. Srinil, G. Rega, Two-to-one resonant multi-modal dynamics of horizontal/inclined cables. Part II: Internal resonance activation, reduced-order models and nonlinear normal modes, Nonlinear Dynamics, 48 (2007) 253-274.

[15] X. Chen, L. Ma, Y. Zheng, D.-W. Lee, Theoretical analysis of postbuckling behavior with experimental validation using electrothermal microbeams, Applied Physics Letters, 98 (2011) 073107.

[16] L. Ruzziconi, A. M. Bataineh, M. I. Younis, W. Cui, and S. Lenci, "Nonlinear dynamics of an electrically actuated imperfect microbeam resonator: experimental investigation and reduced-order modeling," Journal of micromechanics and microengineering, vol. 23, p. 075012, 2013.

[17] A.Z. Hajjaj, A. Ramini, N. Alcheikh, M.I. Younis, Electrothermally Tunable Arch Resonator, Journal of Microelectromechanical Systems, (2017).

[18] [Online].MEMSCAP: http://www.memscap.com/. [19] [Online].Polytec: http://www.polytec.com/us/.

[20] Ouakad, H. M., and Younis, M. I., 2010, "The dynamic behavior of MEMS arch resonators actuated electrically," International Journal of Non-Linear Mechanics, 45(7), pp. 704713.

[21] Younis, M. I., 2011, MEMS linear and nonlinear statics and dynamics, Springer Science \& Business Media. 
[22] A. H. Nayfeh, Introduction to Perturbation Techniques: Wiley, 2011.

[23] M. I. Younis and A. H. Nayfeh, "A Study of the Nonlinear Response of a Resonant Microbeam to an Electric Actuation," Nonlinear Dynamics, vol. 31, pp. 91-117, January 012003.

[24] A. H. Nayfeh, "Resolving controversies in the application of the method of multiple scales and the generalized method of averaging," Nonlinear Dynamics, vol. 40, pp. 61-102, 2005.

[25] J. Barceló, J. Rosselló, S. Bota, J. Segura, J. Verd, Electrostatically actuated microbeam resonators as chaotic signal generators: A practical perspective, Communications in Nonlinear Science and Numerical Simulation, 30 (2016) 316327. 


\section{ANNEX A}

\section{DEFINITION OF COEFFICIENTS}

$$
\begin{aligned}
& \Gamma\left(f_{1}(x, t), f_{2}(x, t)\right)=\int_{0}^{1} \frac{\partial f_{1}}{\partial x} \frac{\partial f_{2}}{\partial x} \mathrm{~d} x \\
& \Lambda_{n m}=2 \alpha_{1}\left(\begin{array}{l}
w_{s}^{\prime \prime}(x) \Gamma\left(\phi_{m}(x), \phi_{n}(x)\right) \\
+\phi_{m}^{\prime \prime}(x) \Gamma\left(w_{s}(x), \phi_{n}(x)\right) \\
+\phi_{n}^{\prime \prime}(x) \Gamma\left(w_{s}(x), \phi_{m}(x)\right)
\end{array}\right) \\
& +\frac{6 \alpha_{2} V_{E f f C} \phi_{m}(x) \phi_{n}(x)}{\left(w_{s}(x)-1\right)^{4}} \\
& \Lambda_{i}=\alpha_{1}\left(\begin{array}{l}
w_{s}^{\prime \prime}(x) \Gamma\left(\phi_{i}(x), \phi_{i}(x)\right) \\
+2 \phi_{i}^{\prime \prime}(x) \Gamma\left(w_{s}(x), \phi_{i}(x)\right)
\end{array}\right)+\frac{3 \alpha_{2} V_{E f f D C} \phi_{i}(x)^{2}}{\left(w_{s}(x)-1\right)^{4}} \\
& R_{1}=\int_{0}^{1} \phi_{m}(x) \Lambda_{n m} \mathrm{~d} x \\
& R_{2}=\int_{0}^{1} \phi_{n}(x) \Lambda_{m} \mathrm{~d} x \\
& F_{2 V D C}=\int_{0}^{1} \frac{\phi_{m}(x) \alpha_{2} V_{A C} V_{D C}}{\left(w_{s}(x)-1\right)^{2}} \mathrm{~d} x \\
& \mu_{i}=\int_{0}^{1} c \phi_{i}(x)^{2} \mathrm{~d} x \\
& F_{2 V A C}=\frac{1}{4}\left(\int_{0}^{1} \frac{\phi_{n}(x) V_{A C}^{2} \alpha_{2}}{\left(w_{s}(x)-1\right)^{2}} \mathrm{~d} x\right)
\end{aligned}
$$

$$
\begin{aligned}
& \mathrm{H}\left[\omega_{i}\right]=H^{i v}-N H^{\prime \prime}-2 \alpha_{1} w_{s}^{\prime \prime}\left(\int_{0}^{1} w_{s}^{\prime} H^{\prime} \mathrm{d} x\right)-\frac{2 \alpha_{2} V_{E f f D C}}{\left(1-w_{s}\right)^{3}} H \\
& -\omega_{i}^{2} H \\
& K_{1}=2 \alpha_{1}\left(\int_{0}^{1} \phi_{m} w_{s}^{\prime \prime} \Gamma\left(\phi_{n}, \psi_{1}\right) \mathrm{d} x\right)+2 \alpha_{1}\left(\int_{0}^{1} \phi_{m} \phi_{n}^{\prime \prime} \Gamma\left(w_{s}, \psi_{1}\right) \mathrm{d} x\right) \\
& +2 \alpha_{1}\left(\int_{0}^{1} \phi_{m} \psi_{1}^{\prime \prime} \Gamma\left(w_{s}, \phi_{n}\right) \mathrm{d} x\right)+2 \alpha_{1}\left(\int_{0}^{1} \phi_{m} \phi_{m}{ }^{\prime \prime} \Gamma\left(\phi_{n}, \phi_{n}\right) \mathrm{d} x\right) \\
& +4 \alpha_{1}\left(\int_{0}^{1} \phi_{m} \phi_{n}{ }^{\prime \prime} \Gamma\left(\phi_{m}, \phi_{n}\right) \mathrm{d} x\right)+4 \alpha_{1}\left(\int_{0}^{1} \phi_{m} w_{s}{ }^{\prime \prime} \Gamma\left(\phi_{m}, \psi_{4}\right) \mathrm{d} x\right) \\
& +4 \alpha_{1}\left(\int_{0}^{1} \phi_{m} \phi_{m}{ }^{\prime \prime} \Gamma\left(w_{s}, \psi_{4}\right) \mathrm{d} x\right)+4 \alpha_{1}\left(\int_{0}^{1} \phi_{m} \psi_{4}{ }^{\prime \prime} \Gamma\left(w_{s}, \phi_{m}\right) \mathrm{d} x\right) \\
& +6 \alpha_{2} V_{E f f D C}\left(\int_{0}^{1} \frac{\psi_{1} \phi_{m} \phi_{n}}{\left(1-w_{s}\right)^{4}} \mathrm{~d} x\right)+12 \alpha_{2} V_{E f(D 12}\left(\int_{0}^{1} \frac{\psi_{4} \phi_{m}{ }^{2}}{\left(1-w_{s}\right)^{4}} \mathrm{~d} x\right) \\
& K_{2}=4 \alpha_{1}\left(\int_{0}^{1} \phi_{m} w_{s}{ }^{\prime \prime} \Gamma\left(\phi_{m}, \psi_{3}\right) \mathrm{d} x\right)+4 \alpha_{1}\left(\int_{0}^{1} \phi_{m} \phi_{m}{ }^{\prime \prime} \Gamma\left(w_{s}, \psi_{3}\right) \mathrm{d} x\right) \\
& +4 \alpha_{1}\left(\int_{0}^{1} \phi_{m} \psi_{3}^{\prime \prime} \Gamma\left(w_{s}, \phi_{m}\right) \mathrm{d} x\right)+3 \alpha_{1}\left(\int_{0}^{1} \phi_{m} \phi_{m}{ }^{\prime \prime} \Gamma\left(\phi_{m}, \phi_{m}\right) \mathrm{d} x\right) \\
& +12 \alpha_{2} V_{E f D C}\left(\int_{0}^{1} \frac{\psi_{3} \phi_{m}^{2}}{\left(1-w_{s}\right)^{4}} \mathrm{~d} x\right)
\end{aligned}
$$

Particular Solutions

$\psi_{1}(x): \mathrm{H}\left[\omega_{n}+\omega_{m}\right]=2 \Lambda_{n m}$

$\psi_{2}(x): \mathrm{H}\left[2 \omega_{n}\right]=\Lambda_{n}$

$\psi_{3}(x): \mathrm{H}[0]=\Lambda_{m}$

$$
\begin{aligned}
K_{3}= & 2 \alpha_{1}\left(\int_{0}^{1} \phi_{n} w_{s}{ }^{\prime \prime} \Gamma\left(\phi_{m}, \psi_{1}\right) \mathrm{d} x\right)+2 \alpha_{1}\left(\int_{0}^{1} \phi_{n} \phi_{m}^{\prime \prime} \Gamma\left(w_{s}, \psi_{1}\right) \mathrm{d} x\right) \\
& +2 \alpha_{1}\left(\int_{0}^{1} \phi_{n} \psi_{1}^{\prime \prime} \Gamma\left(w_{s}, \phi_{m}\right) \mathrm{d} x\right)+4 \alpha_{1}\left(\int_{0}^{1} \phi_{n} \phi_{m}^{\prime \prime} \Gamma\left(\phi_{m}, \phi_{n}\right) \mathrm{d} x\right) \\
& +2 \alpha_{1}\left(\int_{0}^{1} \phi_{n} \phi_{n}^{\prime \prime} \Gamma\left(\phi_{m}, \phi_{m}\right) \mathrm{d} x\right)+4 \alpha_{1}\left(\int_{0}^{1} \phi_{n} w_{s}^{\prime \prime} \Gamma\left(\phi_{n}, \psi_{3}\right) \mathrm{d} x\right) \\
& +4 \alpha_{1}\left(\int_{0}^{1} \phi_{n} \phi_{n}^{\prime \prime} \Gamma\left(w_{s}, \psi_{3}\right) \mathrm{d} x\right)+4 \alpha_{1}\left(\int_{0}^{1} \phi_{n} \psi_{3}^{\prime \prime} \Gamma\left(w_{s}, \phi_{n}\right) \mathrm{d} x\right) \\
& +6 \alpha_{2} V_{E f f D C}\left(\int_{0}^{1} \frac{\psi_{1} \phi_{m} \phi_{n}}{\left(1-w_{s}\right)^{4}} \mathrm{~d} x\right)+12 \alpha_{2} V_{E f f D C}\left(\int_{0}^{1} \frac{\psi_{3} \phi_{n}^{2}}{\left(1-w_{s}\right)^{4}} \mathrm{~d} x\right)
\end{aligned}
$$

$\psi_{4}(x): \mathrm{H}[0]=\Lambda_{n}$ 


$$
\begin{aligned}
K_{4}= & 2 \alpha_{1}\left(\int_{0}^{1} \phi_{n} w_{s} " \Gamma\left(\phi_{n}, \psi_{2}\right) x\right)+4 \alpha_{1}\left(\int_{0}^{1} \phi_{n} w_{s} " \Gamma\left(\phi_{n}, \psi_{4}\right) \mathrm{d} x\right) \\
& +2 \alpha_{1}\left(\int_{0}^{1} \phi_{n} \phi_{n}^{\prime \prime} \Gamma\left(w_{s}, \psi_{2}\right) \mathrm{d} x\right)+4 \alpha_{1}\left(\int_{0}^{1} \phi_{n} \phi_{n}^{\prime \prime} \Gamma\left(w_{s}, \psi_{4}\right) \mathrm{d} x\right) \\
& +2 \alpha_{1}\left(\int_{0}^{1} \phi_{n} \psi_{2}^{\prime \prime} \Gamma\left(w_{s}, \phi_{n}\right) \mathrm{d} x\right)+4 \alpha_{1}\left(\int_{0}^{1} \phi_{n} \psi_{4}^{\prime \prime} \Gamma\left(w_{s}, \phi_{n}\right) \mathrm{d} x\right)(\mathrm{A} .16) \\
& +3 \alpha_{1}\left(\int_{0}^{1} \phi_{n} \phi_{n}^{\prime \prime} \Gamma\left(\phi_{n}, \phi_{n}\right) \mathrm{d} x\right)+6 \alpha_{2} V_{E f f D C}\left(\int_{0}^{1} \frac{\psi_{2} \phi_{n}^{2}}{\left(1-w_{s}\right)^{4}} \mathrm{~d} x\right) \\
+ & 12 \alpha_{2} V_{E f f D C}\left(\int_{0}^{1} \frac{\psi_{4} \phi_{n}^{2}}{\left(1-w_{s}\right)^{4}} \mathrm{~d} x\right) \\
F_{3 V D C}= & 2 \alpha_{2} V_{A C} V_{D C}\left(\int_{0}^{1} \frac{\phi_{m} \phi_{n}}{\left(1-w_{s}\right)^{3}} \mathrm{~d} x\right) \\
F_{3 V A C}= & \frac{\alpha_{2} V_{A C}^{2}}{2}\left(\int_{0}^{1} \frac{\phi_{m}{ }^{2}}{\left(1-w_{s}\right)^{3}} \mathrm{~d} x\right)
\end{aligned}
$$

